\title{
Amping up allergic responses
}

According to the Centers for Disease Control and Prevention, over 22 million people in the United States have asthma. Not all patients respond to commonly prescribed medications, including bronchodilators and anti-inflammatory drugs, which suggests that the disease might be driven by distinct mechanisms in different populations of patients. Current research aims to identify common inflammatory mediators, or cytokines, and self-amplifying loops that drive these inflammatory responses. Drugs that target a particular mediator might dampen the allergic response in a broad range of patients and thereby improve the symptoms of asthma.

T cells are a type of immune cell that is produced in response to allergic challenge. $T$ cells of a particular subset, called Thelper type-2 $\left(\mathrm{T}_{\mathrm{H}} 2\right)$ cells, are commonly found in the lung following allergic challenge, where they promote both inflammation and hyper-responsiveness of the airways. In a recent study (Immunity 43, 318-330; 2015) Bart N. Lambrecht and colleagues from Ghent University (Belgium) examined how $\mathrm{T}_{\mathrm{H}} 2$ cells emerge in the lung following allergic challenge. They sensitized and challenged mice with house dust mites, a common environmental allergen. The frequency of $\mathrm{T}_{\mathrm{H}} 2$ cells increased in response to allergen challenge, as did another type of $\mathrm{T}$ cell that produces the cytokine interleukin-21 (IL-21).

Using transgenic mice in which cells that produce IL-21 were labeled and tracked with green fluorescent protein, the researchers found that these cells first develop in lymph nodes outside the lung before they are found in the lung. This labeling also confirmed that these cells were the major source of IL-21 within the lung.

These T cells, and the IL-21 that they produce, seem to affect several populations of cells in the lung that have been implicated in asthma. Mice that lack the IL-21 receptor show a lower occurrence of $\mathrm{T}_{\mathrm{H}} 2$ cells and eosinophils (another population of cells commonly found in the lungs of asthmatics) following allergic challenge.

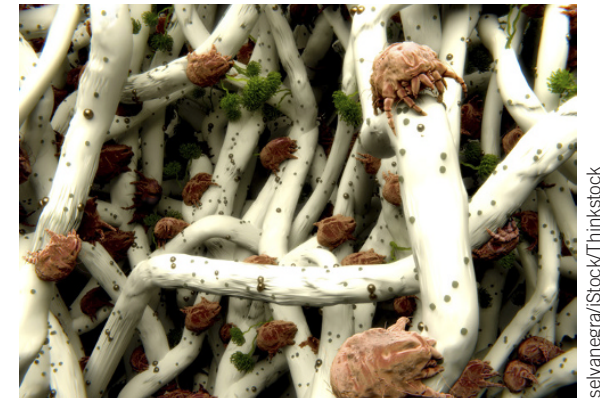

IL-21 seems to amplify the development and recruitment of these cell types to the lung, and when T cells that produce IL-21 are injected into allergic mice they promote the eosinophil accumulation and airway inflammation. IL-21 itself can also promote expansion of $\mathrm{T}_{\mathrm{H}} 2$ cells when injected into mice. Future studies are needed to delineate whether IL-21 acts to sustain the allergic response, and whether it can be targeted in mice with established disease to quell inflammation and reduce hyperresponsiveness in airways.

Kevin Da Silva

\section{TARGETING ION CHANNELS IN CANCER}

Medulloblastoma is the most common malignant pediatric brain tumor and is associated with significant risk of metastasis. Like many cancers, it comes in multiple molecularly defined subgroups. While clinical trials are under way with drugs that treat a quarter of cases that are represented by one molecular subgroup (Sonic hedgehog), targets for drugs have not been identified for the remaining cases. But in a recent paper in Nature Neuroscience (doi:10.1038/nn.4088; published online 10 August 2015), Lily Jan (University of San Francisco) and colleagues showed that a drug targeting a potassium channel was effective in reducing medulloblastoma growth in mice.

Previous work from the Jan group had shown that the voltage-gated potassium channel, EAG2, was more abundant in medulloblastoma cells. Now the authors have shown that mutating the Drosophila homolog, EAG, impaired tumor growth in three different brain tumor models. Curious whether this effect was specific to EAG2, they used gene expression profiling of human medulloblastoma and cells from fly tumors to identify other ion channels whose expression was altered. This allowed them to identify another potassium channel, KCNT2, whose expression is increased in various cancers. Inhibiting expression or activity of KCNT2 also impaired medulloblastoma growth in vitro. Further experiments in medulloblastoma cells showed that both channels regulate cell volume and motility, thus affecting cell growth.

To test whether their findings might have clinical potential, the authors tested several drugs already approved by the FDA to determine whether they might inhibit EAG2 activity and prevent medulloblastoma growth. This screen identified the antipsychotic drug thioridazine. When given to mice with human medulloblastoma cells, thioridazine caused tumor regression.

More than one-fifth of drugs used to treat human diseases target ion channels, but this study is the first to show that such drugs might effectively treat cancer. Further work is needed to determine whether these findings will translate to humans. It is also exciting that an FDA-approved drug could be repurposed to treat cancer, although thioridazine has significant side effects. "The development of a drug designed to act on this newly identified target more specifically and more powerfully, with fewer side effects, would have the potential to improve outcomes for many with this disease," said Jan in a press release. "Targeting of ion channels in cancer is a new frontier, and will be exciting to further explore as a way both to stop the growth of tumors and to limit their potential to spread." 